\title{
Tendencias del Tercer Sector a partir del análisis de las relaciones de interdependencia
}

\author{
Gabriel Vélez Cuartas - Universidad de Antioquia - Departamento de Sociología ${ }^{1}$
}

\section{Resumen}

Este artículo presenta tres tipos de tendencias del Tercer Sector que pueden deducirse de un análisis de redes basado en teorías interorganizacionales y en teoría de dependencia de recursos. A través de dos estudios de caso realizados en la ciudad de Medellín en Colombia, se presentan algunas categorías que se constituyen en tendencias características del sector.

Palabras clave: Tercer sector - análisis ínterorganizacional - análisis de redes sociales - tendencias sectoriales.

\begin{abstract}
This paper presents three different types of tendencies about third sector. This tendencies could be deduce in a network analysis based on interorganizational theories and resource dependence theory. This paper presents two study cases made at Medellin-Colombia. This show some categories that constitute characteristic tendencies of the sector.
\end{abstract}

Key words: Third sector - interorganizational analysis - social network analysis sectorial tendencies.

El Tercer Sector, asociado por lo regular con Organizaciones de la Sociedad Civil, es caracterizado por una gran multiplicidad de intereses y propósitos. Esta caracterización está relacionada con la asunción de principios democráticos otorgados a la sociedad civil: individualidad y libertad inalienables. Así, los presupuestos de una sociedad democrática descansan en la voluntad de esta sociedad civil y su capacidad de elección de su destino, desde Rousseau hasta Habermas.

En la sociedad global actual, estas formas de configuración democrática, antes cobijadas bajo el nombre de pueblo, han tomado distintas formas como evolución de la figura del individuo que se organiza y negocia sus intereses con otros en la sociedad. El pueblo comienza a adquirir rostro en la segunda mitad del siglo XX: movimientos sociales, organizaciones civiles, organizaciones y grupos comunitarios, grupos religiosos, clubes y asociaciones, en fin, toda emergencia organizativa que no tenga un cuño productivo o estatal. A pesar de la transformación, la idea de

\footnotetext{
${ }^{1}$ Enviar correspondencia a: Gabriel Vélez Cuartas, e-mail: gabrielvelezcuartas@yahoo.com.mx
} 
soberanía sigue siendo exclusiva de este sector, por lo tanto el principal motor de la democracia.

Las OSC son tanto prestadoras de servicios como agentes de cambio social y activos participantes en la deliberación pública. Con la prensa libre y la democracia representativa, las OSC constituyen el soporte de las democracias. Estas organizaciones son el canal de acceso directo de la ciudadanía organizada para participar con su voz en el debate público y contribuir en la definición del bienestar colectivo. La movilización de opinión, la promoción de nuevos temas en la agenda pública y la participación en el diseño, implementación y control de política públicas son, entre otras, actividades promovidas por las OSC en las democracias, para promover la equidad y justicia social. Las organizaciones contribuyen con estas actividades a ampliar la representación de la diversidad de intereses sociales y valores colectivos en la arena pública, y ayudan a amplificar la voz de los ciudadanos en la deliberación pública y en el proceso de toma de decisiones. (Villar 2003: 9-10)

No se puede desconocer el papel de las organizaciones civiles al respecto, sin embargo, si se define de manera simple la democracia como las reglas del juego en un sistema político nacional, en donde sus pobladores tienen voz y voto y derecho a la agremiación de intereses para poder proponerlos en la esfera pública, ésta no alcanza a describir las dinámicas de las relaciones entre los distintos miembros del Tercer Sector. Empiezan a adquirir voz y voto no sólo los nacionales propios del sistema político, sino también organizaciones internacionales que tratan de posicionar sus agendas globales; las dicusiones sobre el bien público comienzan a tener características corporativas exclusivas de ciertos sectores de la sociedad que chocan con las propuestas de desarrollo votadas públicamente y asumidas por los representantes; las agendas decididas en reuniones de organismos descentralizados de la ONU o en Foros Mundiales, pueden chocar contra el principio de autodeterminación de las comunidades mismas; la participación comunitaria algunas veces entra en el dilema de seguir los propios designios acordados en la deliberación comunitaria o seguir los lineamientos de planes y proyectos de organizaciones internacionales o gubernamentales para poder financiar sus proyectos; las articulaciones, acciones colectivas o las redes (como espacios de articulación) proclamadas como importantes mecanismos para la participación y la optimización de recursos, se convierten a veces en grupos de discusión con baja incidencia real en políticas públicas; entre muchos otros problemas e interrogantes que surgen de la supuesta profundización de la democracia misma.

De esta forma, mirar al tercer sector, no es sólo ver la democratización de la sociedad en acción, es encontrar las contradicciones del mercado, es observar la lucha de sistemas políticos contrarios (clientelismo versus participación directa, por ejemplo), es hallar yuxtaposiciones en las formas organizativas que generan 
desintegración en las propuestas de desarrollo. Estos no son problemas de desviación de alguna real misión de la sociedad civil con respecto a la democratización, sino características intrínsecas del sector, que también lo determinan.

Estos planteamientos están basados en dos investigaciones realizadas entre los años 2005 y 2006 acerca de las relaciones interorganizacionales de un sector temático como la niñez en el municipio de Medellín, Colombia y otra de relaciones entre organizaciones comunitarias en una de las 16 comunas en las que está dividida la ciudad (la comuna 6 , con una de las más altas densidades poblacionales y con gran capacidad organizativa comunitaria en la ciudad) ${ }^{2}$. Para desarrollar algunas comprensiones sobre la problemática expuesta, se presentarán tres apartados básicos: (1) el enfoque teórico y metodológico aportado como novedoso a la investigación del sector y permite identificar y tipificar estos problemas, (2) las dinámicas relacionales de estas organizaciones y los aportes a la caracterización del sector, (3) algunos comentarios al concepto del tercer sector y conclusiones.

\section{Enfoque teórico y metodológico para observar el Tercer Sector}

Desde los años 70 y en la década de los ochenta, fueron comunes los estudios interorganizacionales (especialmente en EEUU, Francia, Inglaterra y Alemania) para definir las formas de relación entre organizaciones productivas y el Estado y de esa forma observar la construcción de políticas públicas entre otros problemas que ya preveían el concepto de gobernanza moderna (Turk, 1970; Knoke, 1986; Le Galès et al., 1995, entre otros). Estos estudios se han basado en gran parte en los desarrollos de la sociología organizacional, especialmente propuesta por autores como Pfeffer y Salancik (1978) desde la teoría de dependencia de recursos y otros autores que se dedicaron a implementar estos principios en las relaciones interorganizacionales.

\footnotetext{
2 Estas dos investigaciones se constituyeron con dos objetivos generales específicos: la primera, construir un mapa de las relaciones interorganizacionales que atienden de alguna u otra forma la niñez en Medellín, para tratar de develar posibilidades de integración o movilidad de recursos a favor de la niñez. La segunda investigación intenta construir un mapa de relaciones de todas las organizaciones comunitarias de una de las comunas de Medellín (la 6, Medellín está dividido en 16 comunas), con el propósito de evaluar los procesos de planeación estratégica y el papel de la participación y la movilización de recursos con respecto a ésta. Este documento, pretende tomar ambos casos y describir algunas tendencias de las organizaciones de la sociedad civil a partir de las estructuras en las que están inmersas. A ambos estudios fueron aplicados los mismos recursos teóricos y metodológicos y a poblaciones organizacionacionales con características similares, lo que permite una contrastación y la ubicación de tendencias en el sector. La contrastación se hace suponiendo ambos estudios como casos específicos del comportamiento del tercer sector en una localidad específica y con expectativas de desarrollo con respecto a su mismo objeto local.
} 
Como se dijo anteriormente, estos estudios han sido especialmente diseñados para organizaciones productivas y la administración pública, sin embargo el terreno de las organizaciones de la sociedad civil ha sido algo relegado en este tipo de investigaciones. Esta circunstancia ha dejado un gran vacío a la construcción de conocimiento acerca del Tercer Sector, en tanto se pierden esferas de análisis que se pueden desprender de nuevos datos sobre su realidad.

Sin embargo no se pueden desconocer algunos estudios y escritos recientes en Latinoamérica desde la perspectiva ínterorganizacional y el policy networks (Sulbrand, Lira e Ibarra, 2001; Porras, 2000). O los realizados en Colombia por Palacio, Hurtado y Garavito (2003) o Vélez (2006) y Brand et. Al (2007).

Las investigaciones más relevantes en el campo han estado dedicadas a observar de un lado el fortalecimiento de las organizaciones civiles (Salamon, 1999; Acotto, 2003; entre otros) y los movimientos sociales, así como las relaciones entre la administración pública y el tercer sector desde estudios políticos e incidencia en las agendas públicas (González y Villar, 2003; Cravacuore, Daniel, Sergio Ilari y Alejandro Villar, 2004) o la concertación para el desarrollo local (Rofman y Villar, 2006; Forni y Longo, 2004; Natal, 1998). También han abundado estudios sobre la participación y el desarrollo del poder local. Sin embargo ha sido descuidado el campo como tal de las relaciones, los intercambios, las dependencias y los tipos de alianzas generados dentro del tercer sector mismo, lo cual se convierte en pieza fundamental para tratar de entender las dimensiones y características de los tipos de desarrollo que están construyendo, más allá del debate de la participación o no en las políticas públicas, pues con o sin políticas públicas a su favor, las organizaciones de la sociedad civil muchas veces construyen desarrollo desde visiones particulares, distintas a las de los propios gobiernos locales. Esto implica que la participación no puede ser vista únicamente como un mecanismo de relación con el Estado o un proceso de empoderamiento en la esfera pública, sino como un proceso social del cual pueden surgir diversas alternativas que pueden chocar con las mismas políticas públicas o potenciarlas.

La teoría de dependencia de recursos y el análisis ínterorganizacional, tienen básicamente un principio, y es que una organización existe porque entra a participar en un juego de interdependencia. En otras palabras, una organización necesita recursos para operar y la única forma de obtenerlos, es a través de negociaciones con otras organizaciones para alcanzar su provisión. Pero no es sólo un problema de provisión, sino también de juegos de poder, en tanto las organizaciones juegan intentando obtener una mayor control sobre los recursos escasos, sea a través de la posesión, acaparamiento, o inclusive la lucha por la 
democratización de algunos que están en manos de monopolios u oligopolios a los cuales tienen acceso pocas organizaciones (Pfeffer y Salancik, 1978).

Este principio aplicado al Tercer Sector, implica un aporte novedoso al centro de las preocupaciones. El desarrollo organizacional, desde este punto de vista, dependerá más de las relaciones que se puedan tejer entre un grupo grande o reducido de organizaciones, que de las disposiciones internas de cada organización y sus características ideológicas, estructurales o funcionales. Gana importancia la forma en que las organizaciones sortean las interdependencias y logran tomar decisiones que no sólo beneficien el crecimiento de la organización, sino también el logro de sus objetivos (ideológicos, prácticos, políticos o económicos).

En el manejo de interdependencias se pueden identificar estructuras de relaciones que se establecen con este fin. Esas estructuras emergentes, por lo regular describen resultados de negociaciones y alianzas entre distintas organizaciones, que dependiendo del tamaño de la estructura o la aparición de múltiples estructuras o subgrupos dentro de una localidad. Pueden identificarse no sólo posiciones con respecto a diferentes perspectivas de desarrollo, sino también la integralidad e integración que pueden estar teniendo las organizaciones en una localidad específica para evaluar su desempeño. En otras palabras, es posible hallar estructuras muy interconectadas que aparentemente pueden estar trabajando de forma integral e integrada, o fragmentos de estructura que denotan fragmentación y desacuerdos con respecto a las perspectivas de atención e intervención en lo social.

En las estrategias tomadas para sortear la interdependencia se pueden identificar otro tipo de factores que surgen de las relaciones de intercambio establecidas: afinidades temáticas o alianzas que se establecen a partir de la especialización de las mismas organizaciones; afinidades en cuanto a posiciones ideológicas o discursivas; alcances no sólo de las relaciones, sino también de capacidad de alcance territorial; y formas del desarrollo del capital social de las organizaciones mismas (en cuanto al concepto de capital social aplicado a las organizaciones vease Lazega, 2004).

Es en este punto donde entra el análisis de redes sociales como metodología. La mezcla de un análisis ínterorganizacional basado en la teoría de dependencia de recursos y el análisis de redes, permite no sólo observar centralidades, cliques, pertenencias o afiliaciones, puntos de quiebre, puentes y componentes, sino también recursos abundantes y escasos, proveedores y beneficiarios, sectores que controlan la ejecución de proyectos (por tanto perspectivas de desarrollo aplicadas) 
y características de las acciones colectivas (para el desarrollo de este concepto, véase Elster, 1991; Olson, 1965).

De esta forma la toma de decisiones de las organizaciones de la sociedad civil, queda determinada no sólo por la coherencia con respecto a los propósitos de las organizaciones, sino también por más posibilidades: intercambio económico, participación desde la deliberación y representación de intereses. El intercambio económico define las interdependencias, pero también representaciones y alianzas, así como los tipos de aliados en la implementación de agendas y la forma de construcción de las agendas desde el punto de vista de lo que se financia o lo que no se financia y quiénes lo financian (lo que lleva a mirar los grados de integración o no de las agendas). La participación y la deliberación enuncian los debates, las posiciones políticas, los alcances de las agendas (en cuanto a los tipo de actores que concurren a los espacios de deliberación y decisión política y práctica) y la fragmentación o no del horizonte de la localidad en cuanto a acuerdos, duplicación de esfuerzos, multiplicidad de visiones y frentes de acción en conflicto o sinergia, inclusive la capacidad $o$ no de influencia en las políticas públicas o su distanciamiento de éstas.

En definitiva se propone una teoría intermedia, proveniente de la sociología organizacional, como herramienta interpretativa de los resultados provenientes de un análisis de redes sociales exploratorio. Esto implica definir el desempeño del Tercer Sector desde una perspectiva relacional y estructural. El Tercer Sector no está sólo y es relevante el papel que juegan en su configuración los otros sectores. De allí que la definición del término tercer sector tenga que revisarse a la luz de elementos relacionales y no sólo por las características intrínsecas de su constitución como se haría desde una teoría organizacional clásica (ver definición de tercer sector, Salamon et al., 1999) ${ }^{3}$.

\footnotetext{
${ }^{3}$ La definición de Salamon et al. “Organizaciones: es decir, poseen una presencia y una estructura institucionales; privadas; tienes existencia separada a la del Estado; no reparten beneficios: no generan beneficios para sus gestores o el conjunto de titulares de las mismas; autónomas: controlan esencialmente sus propias actividades; con participación de voluntarios: la pertenencia a ellas no está legalmente impuesta y atraen un cierto nivel de aportaciones voluntarias de tiempo o dinero" (1999:20).
} 


\section{Dinámicas de relación y Tercer Sector diverso}

En los dos estudios presentados (Vélez:2006; Brand et al:2007) ${ }^{4}$ se han identificado tres tipos de recursos básicos intercambiados entre las organizaciones: recursos económicos y de cooperación, información y conocimiento y capital social acumulado entre las organizaciones a través de afiliaciones a espacios de articulación. Estos tres tipos de recursos fueron estudiados en los intercambios entre organizaciones comunitarias de la comuna 6 de Medellín en el caso del estudio de Brand et al., y entre todas las organizaciones (gubernamentales y no gubernamentales que atienden a la niñez en la ciudad. Para el primer estudio se identificaron 133 organizaciones las cuales fueron encuestadas, las cuales identificaron relaciones con 457 organizaciones de distintos sectores (Brand et al. 2007:72). En el segundo estudio se identificaron en la ciudad 128 organizaciones que trabajan directamente con la niñez, las cuales dieron cuenta de 455 organizaciones de distintos sectores con las que se relacionan ${ }^{5}$.

Este artículo se propone hacer una contrastación con algunos indicadores simples del análisis de redes y algunas características organizacionales. En esta comparación se lograron identificar tres categorías que se presentan como tendencia en el tercer sector: una tendencia a una separación entre organizaciones temáticas y otras territoriales; una tensión entre la autonomía y la heteronomía; y una tendencia a cierta articulación fragmentada con respecto a una visión

\footnotetext{
${ }^{4}$ Los dos estudios en los que se basa este artículo han utilizado la metodología de la bola de nieve para construir su universo. Se acudieron a múltiples bases de datos que describían organizaciones formalmente constituídas que trabajaran con la niñez, igual que para el segundo caso. Las relaciones que estas organizaciones referenciaron en conjunto con ellas, constituyeron el universo total de la investigación. Para la de niñez se detectaron alrededor de 508 organizaciones gubernamentales, de la sociedad civil y del mercado, de estas se encuestaron 128 organizaciones gubernamentales y de la sociedad civil (el resto no atendían propiamente a la niñez o no se consiguieron suficientes datos para involucrarlas directamente como organizaciones con programas para este sector poblacional). Para la de Comuna 6, 458 organizaciones, de las cuales fueron encuestadas 133 (las propiamente comunitarias, el resto son ONG, organizaciones gubernamentales o del mercado) Si bien para el estudio del Sistema de Atención a la Niñez de Medellín, no está la totalidad de organizaciones, sí están las más visibles por la cantidad de recursos invertidos y con los programas más visibles en la ciudad (las no incluidas o fueron agregadas en las organizaciones que las rigen, por ejemplo las escuelas públicas fueron agregadas en la Secretaría de Educación Municipal, o puede presumirse un trabajo con poco impacto por su aislamiento de estas estructuras centrales propuestas). Las encuestas fueron aplicadas en su mayoría a personas encargadas de programas y proyectos en las organizaciones o alguna de sus directivas, dependiendo de la disponiblidad. Ambos estudios son de carácter descriptivo y utilizan el análisis de redes sociales a partir de categorías interorganizacionales.

${ }^{5}$ La comuna 6 de Medellín es la más poblada de la ciudad según el anuario estadístico del año 2005. En ella habitan $192.656 \quad$ personas. http://www.medellin.gov.co/alcaldia/jsp/modulos/N_admon/index.jsp?idPagina=762 el 6 de marzo de 2007. El mismo anuario dice que Medellín está habitado por 2'093.624 personas. Es importante también resaltar que ninguno de los dos estudios anteriores se tuvieron encuenta organizaciones educativas ni de salud, las cuales superan en gran número las presentadas en estos estudios. La mayoría de éstas pertenecen al gobierno.
} 
panorámica del desarrollo local. Se utilizan medidas simples de contrastación, no por disminución de la complejidad para el análisis, sino porque precisamente gracias a los análisis complejos realizados en los estudios, se pudo simplificar la complejidad ínterorganizacional para descubrir algunas tendencias. A continuación se presenta una tabla previa en donde se distribuyen las tendencias identificadas, las categorías empleadas para el análisis y su propósito. Estas serán desarrolladas en los próximos numerales ${ }^{6}$.

\begin{tabular}{|c|c|c|}
\hline Categoría & Herramienta analítica & Propósito \\
\hline \multirow[t]{3}{*}{$\begin{array}{l}\text { Territorial/ } \\
\text { Temático }\end{array}$} & $\begin{array}{l}\text { Inclusión y exclusión de estructuras de } \\
\text { intercambios de recursos (económicos, } \\
\text { cooperación e información }\end{array}$ & $\begin{array}{l}\text { Observar la existencia o no de } \\
\text { intercambios entre el sistema de } \\
\text { atención a la niñez y las } \\
\text { organizaciones comunitarias de la } \\
\text { Comuna } 6\end{array}$ \\
\hline & $\begin{array}{l}\text { Exploración visual de la densidad de las } \\
\text { estructuras de intercambio y número de } \\
\text { relaciones en contraste con número de } \\
\text { organizaciones }\end{array}$ & $\begin{array}{l}\text { Observar la cantidad de flujos de } \\
\text { recursos }\end{array}$ \\
\hline & Co-pertenencias a espacios de articulación & $\begin{array}{l}\text { Revelar exclusiones e inclusiones } \\
\text { entre comunitarias y ONG } \\
\text { (territoriales y temáticas) }\end{array}$ \\
\hline \multirow[t]{2}{*}{$\begin{array}{l}\text { Autonomía/ } \\
\text { heteronomía }\end{array}$} & $\begin{array}{l}\text { No. de relaciones de intercambio de } \\
\text { recursos entre organizaciones }\end{array}$ & $\begin{array}{l}\text { Observar control sectorial y } \\
\text { organizacional de recursos } \\
\text { Observar contingencias en la } \\
\text { planeación del desarrollo local }\end{array}$ \\
\hline & $\begin{array}{l}\text { Grado de modernización a través de las } \\
\text { formas de planeación y posibilidades de } \\
\text { consecusión de recursos, formación } \\
\text { profesional }\end{array}$ & $\begin{array}{l}\text { Observar posibilidades de generar } \\
\text { autonomías por autosuficiencia } \\
\text { organizacional en la planeación }\end{array}$ \\
\hline \multirow[t]{2}{*}{$\begin{array}{l}\text { Articulación/ } \\
\text { fragmentación }\end{array}$} & $\begin{array}{l}\text { Pertenencias o afiliaciones y co-pertenencias } \\
\text { a espacios de articulación }\end{array}$ & $\begin{array}{l}\text { Observar las dinámicas de } \\
\text { espacios de articulación en cuanto } \\
\text { a inclusión y exclusión }\end{array}$ \\
\hline & $\begin{array}{l}\text { Abundancia de espacios de articulación y } \\
\text { alcances en términos de convocatoria }\end{array}$ & $\begin{array}{l}\text { Observar la contingencia del } \\
\text { desarrollo local a partir de la } \\
\text { fragmentación de planeación y } \\
\text { acciones supuestamente } \\
\text { articuladas }\end{array}$ \\
\hline
\end{tabular}

Tabla 1. Resumen de las categorías, herramientas y objetivos de la investigación

\subsection{Lo territorial y lo temático en el tercer sector}

En un primer vistazo de los grafos de ambas investigaciones en los intercambios de recursos económicos, cooperación, información y en las afiliaciones a espacios de articulación (comités, consejos, redes, asociaciones, etc.), se puede observar que los componentes de la comuna 6 (que tienen un referente territorial y local para la acción), incluyen muy pocas organizaciones del sistema de atención a la niñez

\footnotetext{
${ }^{6}$ En este texto se presentan algunos datos emergentes del análisis, para observar el desarrollo analítico completo remitirse a los dos estudios publicados en la página redes (www.redes-sociales, net).
} 
(excepto algunas pocas gubernamentales y cajas de compensación); en tanto el sistema de atención a la niñez, incluye algunas pocas de las más fortalecidas en organización formalizada y larga trayectoria de la comuna 6. Esto comienza a hacer evidente una brecha entre las preocupaciones de tipo territorial y los intereses temáticos (en este caso poblacional: la niñez). Esto puede querer decir que las organizaciones pueden organizarse para prestar servicios o generar procesos de desarrollo de dos formas distintas y no articuladas de manera fuerte: una tendencia de orientación hacia las problemáticas territoriales (del barrio, la zona o la comuna) y otra tendencia hacia la atención de problemas de carácter temático (agendas especializadas en temáticas del desarrollo), más allá de los territorios en los que se pueden desarrollar. Por un lado la organización comunitaria y por el otro lado las organizaciones de la sociedad civil prestadoras de servicios de carácter público.

Esta diferencia ya se advierte en Crovacuore cuando describe algunos casos de participación en Argentina:
Aunque en el pasado se generaron experiencias aisladas de articulación entre gobiernos locales y organizaciones de la sociedad civil y empresas, es a partir de los 90 que comienzan a generalizarse en Argentina los planes estratégicos participativos en municipios y los consejos consultivos, tanto temáticos como de carácter territorial. Entre los consejos temáticos se distinguen los consejos municipales que se crearon en distintas jurisdicciones para fortalecer a los grupos vulnerables (mujeres, niños, discapacitados). A nivel territorial, vale decir, considerando a los barrios como ámbito de participación, una experiencia novedosa en la Provincia de Buenos Aires fue la de los Consejos de la Comunidad, desarrollados en el municipio de Lomas de Zamora desde 1992. (Cravacuore 2004:45)

La diferencia con respecto a los planteamientos de Cravacuore, está en los grados de articulación de estos dos niveles de planeación. La articulación aquí se mediría en el número de relaciones de intercambio entre ellos dos y la inclusión de unas en el sistema de las otras. Por ejemplo, sólo se identificaron 5 organizaciones comunitarias, de las 133 encuestadas en la comuna 6, como prestadoras de servicios a la niñez, en tanto que sólo 4 organizaciones no comunitarias (de las 128 encuestadas) dijeron prestar algún tipo de servicio focalizado para la niñez de la comuna ${ }^{7}$.

En cuanto a las relaciones entre las organizaciones del sistema de atención a la niñez de Medellín y las organizaciones comunitarias de la 6 , se pudieron observar:

\footnotetext{
${ }^{7}$ Se debe tener en cuenta que la administración municipal hace presencia en la comuna y con múltiples programas, pero de las organizaciones gubernamentales encuestadas, no se pudo conseguir datos precisos de focalización en este estudio, que no quiere decir que no existan.
} 
- En recursos ecónomicos en el sistema de atención a la niñez, las comunitarias de la comuna 6 que dijeron participar de este sistema reciben recursos especialmente de organizaciones internacionales y la Alcaldía que pertenecen al sistema de niñez, aunque gran parte de sus recursos también provienen de autofinanciación sea a través de actividades programadas para recoger recursos o por el voluntariado mismo de las personas de las comunidades. Esto da un total de alrededor de 12 organizaciones relacionadas con la comuna 6 desde el sistema de atención a la niñez: 6 gubernamentales, 4 internacionales, 1 comunitaria y 1 fundación. En cuanto a la red ínterorganizacional de la comuna 6 se pueden destacar organizaciones que ofrecen recursos y también hacen parte del sistema de atención a la niñez ${ }^{8}$ : 13 estatales (con 32 relaciones), 2 cajas de compensación ( 3 relaciones) y 3 internacionales ( 3 relaciones). Estas 38 relaciones de intercambio de recursos, representan casi la mitad de la financiación del sistema que son 80 relaciones.

- En cooperación sólo dos organizaciones comunitarias de la comuna, reciben algún tipo de cooperación dentro del sistema de atención a la niñez de Medellín, una de ellas es de otra organización comunitaria de la comuna y la otra de organizaciones de la misma comuna (que son nombradas también en el estudio de la comuna 6). En tanto sólo una ofrece a otra organización de la sociedad civil no comunitaria y la otra ofrece cooperación a otra comunitaria de la misma comuna. En cuanto a las relaciones de cooperación en el estudio de la comuna se detectaron 167 relaciones de cooperación y sólo una pocas con 2 cajas de compensación que están dentro del sistema de atención a la niñez, 6 con organizaciones estatales, 1 con una organización comunitaria por fuera de la comuna.

- En información las mismas 5 organizaciones comunitarias que dijeron trabajar directamente en la comuna 6 y que pertenecen al sistema de atención a la niñez, más dos espacios de articulación (a los que pertenecen también estas organizaciones), reciben información de organizaciones del Sistema. Estas organizaciones son 4 organizaciones de la sociedad civil no comunitarias, 10 comunitarias de otras comunas de la ciudad, 3 espacios de articulación ( 1 de la comuna), 1 comunitaria de la zona, 4 del gobierno y 4

\footnotetext{
${ }^{8}$ Aunque sean las mismas organizaciones no necesariamente están articulando programas de niñez con atención a programas territoriales de la comuna, pues pueden responder a distintos objetivos los proyectos financiados, lo que se demuestra al no haber sido nombradas algunas de estas organizaciones en el sistema de atención a la niñez.
} 
internacionales. En el estudio de las relaciones interorganizacionales de la comuna, sólo una organización de la sociedad civil no comunitaria que aparece en el sistema de atención a la niñez se presenta como donadora. Es importante anotar que el resto son organizaciones comunitarias de la misma comuna. Esta información en su mayoría va a dar las mismas organizaciones comunitarias, algunas pocas comunitarias de otras comunas, y dos o tres del gobierno.

- En co-pertenencia a espacios de articulación, sólo una pertence a 7 espacios de articulación centrales en el sistema de atención a la niñez y otras dos más dijeron pertenecer a uno o dos espacios de articulación no tan centrales para el sistema. Las otras 2 no dijeron pertenecer a algún espacio de articulación. En tanto a las afiliaciones a espacios de articulación en la comuna, se pudieron detecatar alrededor de 10 participaciones en espacios convocados por organizaciones del gobierno que participan también en el sistema de atención a la niñez (claves para la planeación participativa y el desarrollo de la comuna, pero no necesariamente dedicados a la niñez), 1 convocado por una organización comunitaria y otro por organización civil no comunitaria que coordina un importante espacio para la participación en el diseño e implementación de políticas públicas para la niñez (la Red Antioqueña de Niñez).

Excepto en el punto de la financiación de programas de la comuna 6 (se contaron la mitad de relaciones sostenidas con organizaciones que pertenecen al sistema de atención a la niñez), las otras relaciones entre sistema de atención a la niñez de Medellín y estructura de relaciones interorganizacionales comunitarias son débiles. Hay pocas organizaciones que pueden servir de puente entre lo territorial y lo temático de la niñez. Algunas acotaciones: las 38 relaciones de financiación provienen en su mayoría de organizaciones del gobierno. Estas deben atender gran cantidad de problemáticas en su agenda y estas mismas organizaciones se fragmentan en diversas unidades (no conectadas entre sí operativamente por lo regular) que pueden atender la temática de niñez por un lado y por el otro, preocuparse de problemas territoriales y no temáticos. Por otra parte, sólo se registraron 80 relaciones de financiación a proyectos comunitarios, entre las 133 organizaciones encuestadas. Esto denota un alto grado de trabajo voluntario por parte de las organizaciones que no dijeron tener ningún tipo de financiación.

De otro lado es importante resaltar que existe un alto intercambio de información entre las organizaciones de la comuna, pero hacia fuera, pareciera ser muy deficiente, especialmente con respecto al tema de niñez. Se crean pues 
centralidades en unas pocas organizaciones que pueden conectar sistema de niñez y estructura relacional de la comuna (las 5 mencionadas anteriormente).

No se propone la articulación como imperativa, pero si se trata de generar un horizonte integral e integrado en el desarrollo, se destacan dos tendencias (territorial y temática) no articuladas y orientadas al azar, lo que implica encuentros o desencuentros fortuitos, no planeados. Esto complejiza la generación de acuerdos frente a las prioridades de atención en los territorios mismos. No se trata sólo de usuarios de servicios, sino también de comunidades que tienen sus propias perspectivas del desarrollo construidas de manera participativa. De esta forma se genera yuxtaposición de programas en un mismo territorio, que no orientan sus acciones de manera articulada.

El tercer sector no sólo presenta características organizacionales intrínsecas, sino también tendencias en vías distintas: territorialización y tematización. Es importante aclarar que esto no responde a una generalización, sino a la detección de una tendencia que puede presentarse en diferentes localidades.

\subsection{Autonomía y heteronomía otras dos tendencias distintas}

En las organizaciones modernas hay una fuerte tensión entre conservar la autonomía y ajustar la heteronomía de tal forma que puedan coincidir los intereses propios con los de quienes tienen los recursos que necesitan para la ejecución de sus proyectos. El ajuste de la heteronomía puede conducir por tres vías: una, encontrar financiadores que coinciden completamente con los propósitos de la organización que se presenta; dos, encontrar coincidencias parciales y lograr negociaciones que ceden de un lado y otro; tres, no encontrar afinidades, por lo tanto generar una cooptación por parte del organismo financiador, sino hay fuentes suficientes. Una última opción es desaparecer o crear una organización distinta.

En estos ajustes a la interdependencia o heteronomía, se pueden observar algunos contrastes entre los dos casos presentados. A continuación se presentan los gráficos de intercambios económicos en ambos: 

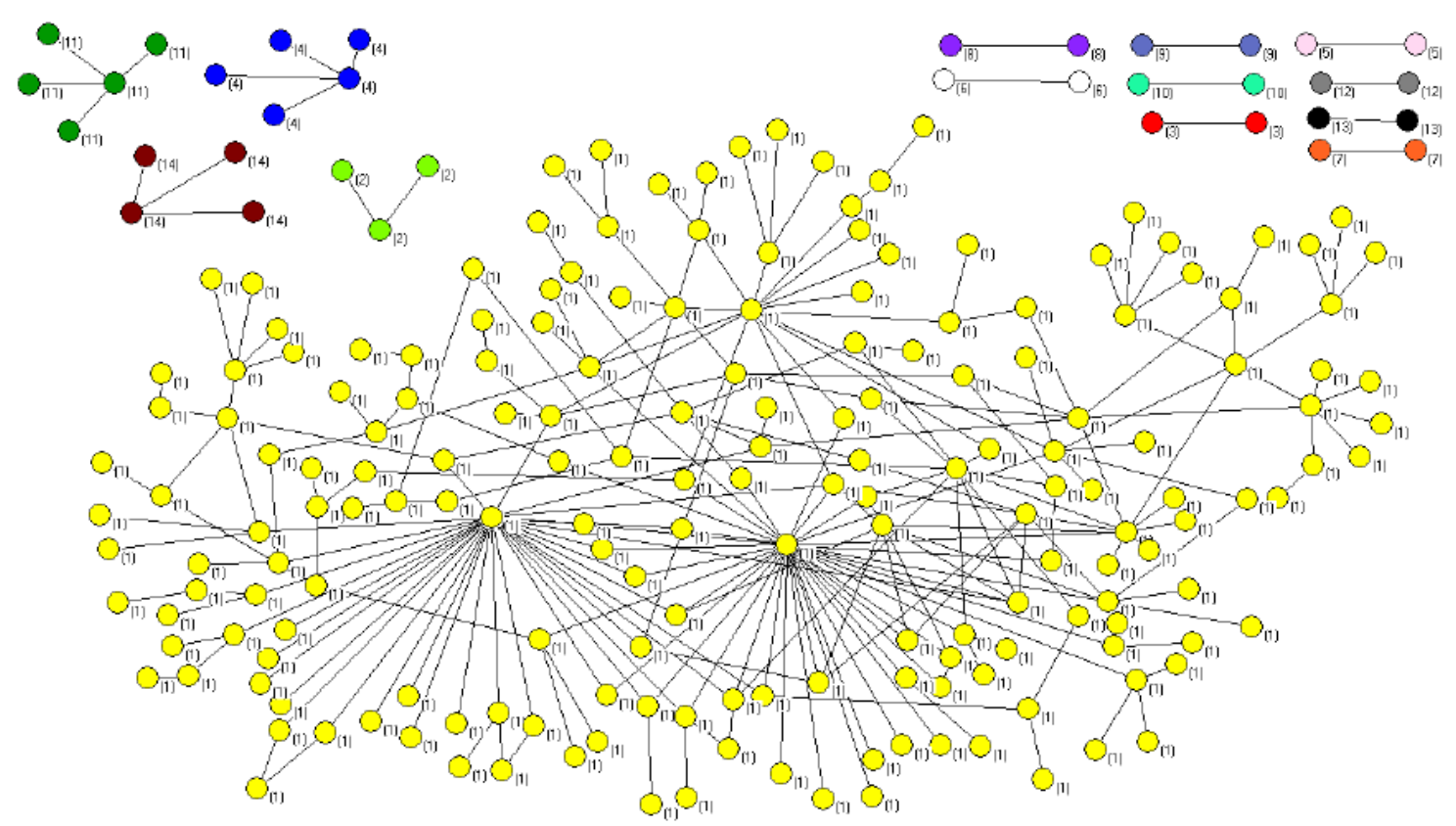

Figura 1. Gráfico de relaciones de intercambio de recursos económicos: Sistema de Atención a la Niñez de Medellín (tomado de Vélez 2006: 113).

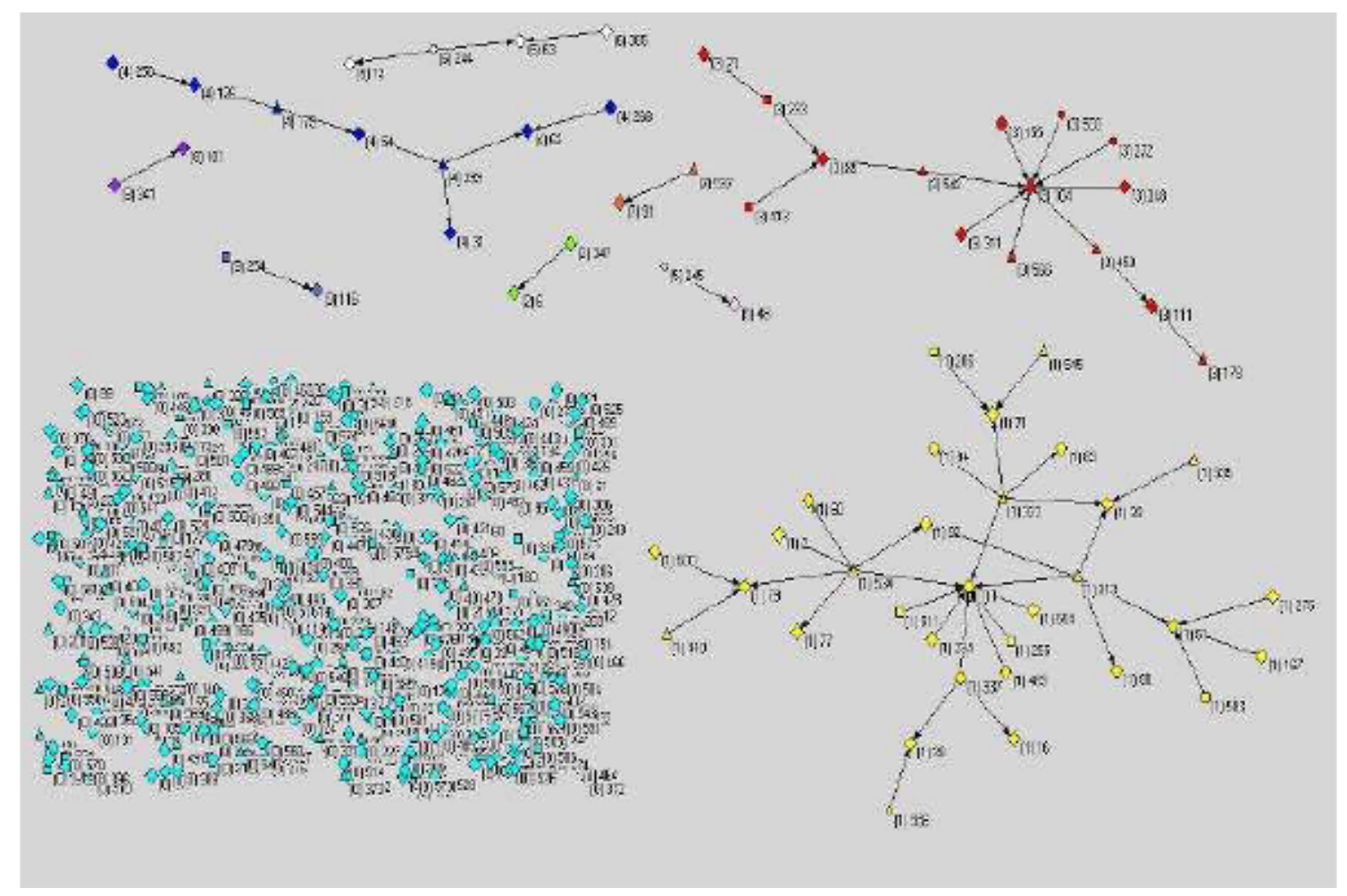

Figura 2. Gráfico de intercambio de recursos económicos comunitaria comuna 6 (tomado de Brand et al. 2007: $\left.100^{9}\right)$.

${ }^{9}$ Ambas gráficas fueron construidas con el software PAJEK. Batagelj V., A. Mrvar: Pajek-Program for Large Network Analysis. Home page: http://vlado.fmf.uni-lj.si/pub/networks/pajek/ 
Los colores en los puntos de las dos redes representantes componentes. En la primera red (sistema de niñez) 128 organizaciones dijeron tener 330 relaciones de financiación, en tanto que en la segunda, las 133 organizaciones sólo reconocieron 80. También se destaca a simple vista una mayor densidad en las relaciones en el sistema de atención a la niñez que en el ámbito territorial de la comuna 6 . Esto puede ocurrir por la presencia de por lo menos dos fenómenos simultáneos o que se presentan de manera individual:

1) Que hay mayor financiación disponible para problemas temáticos según la orientación de los organismos internacionales que financian, algunas organizaciones gubernamentales y la misma orientación de las organizaciones que se autofinancian. Debido a la multiplicidad de fuentes de financiación, estas organizaciones probablemente puedan, desarrollar una autonomía mucho mayor que las mismas organizaciones comunitarias, entre las cuales escasean las relaciones de financiación. La relativa autonomía, da a las organizaciones el poder de negociar de forma más horizontal con las organizaciones que son financiadoras, por la diponibilidad de múltiples fuentes de ingresos. Esto hace que las organizaciones sin financiación o fuentes posibles, más fácilmente declinen ante sus propósitos comunitarios o las ponga en conflicto entre intereses temáticos y territoriales.

2) Sin embargo hay un factor que puede ser más importante y es el grado de modernización de las organizaciones mismas. Hablar de modernización, es encontrar en las organizaciones infraestructura para la planeación, la gestión de recursos y la especialización de funciones. Mientras más estructurada sea una organización en estos términos, más fácilmente puede acceder a recursos y presentar proyectos a todo tipo de organizaciones que requieren de cierta formalización para poder desembolsar los recursos con un mínimo nivel de garantía.

La mayoría de las organizaciones comunitarias evalúan y planean, sin embargo su infraestructura es frágil y depende de las voluntades, no pueden garantizar puestos fijos, como tampoco salario a sus trabajadores (que además tienen que trabajar en otras organizaciones para vivir). Además hay pocas personas capacitadas con un grado universitario (no necesariamente el liderazgo se basa en los grados educativos, pero sí las destrezas técnicas de administración y diseño de programas y proyectos). Esto hace que no existan las mismas condiciones competitivas para lograr el acceso a un recurso por parte de las organizaciones territoriales (comunitarias), que las especializadas en un campo temático como la niñez, las cuales tienen una constitución formal y gran experiencia en captación de recursos a 
través de estrategias, incluyendo la planeación formal. Las experiencias comunitarias logran consolidación en el tiempo sólo cuando estas se institucionalizan y mejoran su infraestructura, como ocurre con las 5 que participan del Sistema de Atención a la Niñez y trabajan directamente en la comuna 6, y algunas otras pocas. Esto no quiere decir que las otras no cumplan alguna función, pues responden al fortalecimiento de las relaciones comunitarias y a la atención de eventualidades importantes para la comunidad misma, así como generando ciertos ejercicios participativos útiles para procesos como Presupuestos Participativos o la relación directa con el gobierno como interlocutores como las Juntas de Acción Comunal.

Precisamente esta diferencia funcional, genera distintas tendencias en las formas de construcción de autonomía de las organizaciones. Por un lado está la tendencia a la modernización de las organizaciones que les genera capacidad de captación de recursos y mayores potenciales en la formulación de proyectos. Las autonomías aquí se definen por afinidades en perspectivas temáticas (o perspectivas de atención a la niñez en nuestro caso). Por otro lado las comunitarias, si bien parecieran tener una heteronomía más acentuada por su escasez de recursos, esta escasez se convierte en potencial de articulación en tanto genera procesos de participación comunitaria importantes en la toma de decisiones. Habría un tercer tipo de tendencia y es la más conflictiva en tanto algunas organizaciones comunitarias alcanzan ciertos niveles de modernización lo cual las pone entre el entrar a competir con otras organizaciones de carácter temático por recursos, y el no salirse de su misión comunitaria más heterogénea que lo temático especializado. Estas organizaciones necesitan especializarse en varios frentes y sortear la dependencia de recursos que surge a partir de la multiplicación de labores y requerimiento de personal.

Los tres tipos de tendencias en la configuración organizacional resultantes, serían: una que se caracteriza por organizaciones informales, emergentes dependiendo de las problemáticas e impulsoras de la participación directa y el fortalecimiento de los lazos; otra que se caracteriza por la modernización de su infraestructura y formalización de sus mecanismos organizacionales permitiendo una alta especialización de funciones dentro de la organización misma (administración, gestión interna y externa, ejecución de proyectos); la tercera se caracteriza por un híbrido entre modernización y sostenimiento de procesos comunitarios alternos a la misma organización de carácter más informal. Este tipo de organizaciones aunque altamente funcional por las posibilidades de conectar grupos numerosos de 
organizaciones, se ven enfrentadas constantemente a conflictos entre el mercado y el mundo político de la participación.

En cuanto a la autonomía y a la heteronomía, se puede detectar una tendencia más y es al aumento de contingencia en la puesta en práctica del desarrollo local. La planeación del desarrollo repartida en un gran número de organizaciones, que a veces logran generar acuerdos y a veces no, hacen que los efectos de una intervención de una organización determinada puedan ser anulados por la intervención de otra, generando efectos contrarios a los deseados. O tal vez coincidencialmente logren potenciar el proyecto de unos y otros. Algunas cifras pueden ser ilustrativas de estas autonomías logradas por las organizaciones.

En el Sistema de Atención a la Niñez de Medellín se pueden identificar los siguientes flujos de financiación entre sectores organizacionales:

Tabla 31 de flujos de financiación entre sectores

\begin{tabular}{|l|l|r|}
\hline Emisora & Receptora & $\%$ \\
\hline Organizaciones gubernamentales & Organizaciones gubernamentales & 97,2 \\
\hline Organizaciones de la sociedad civil & Organizaciones gubernamentales & 1,2 \\
\hline Sin dato sobre sector & Organizaciones gubernamentales & 1,6 \\
\hline Organizaciones internacionales & Organizaciones gubernamentales & 0 \\
\hline Organizaciones gubernamentales & Organizaciones de la sociedad civil & 27,3 \\
\hline Organizaciones de la sociedad civil & Organizaciones de la sociedad civil & 45,4 \\
\hline Organizaciones internacionales & Organizaciones de la sociedad civil & 10 \\
\hline Sin dato sobre sector & Organizaciones de la sociedad civil & 17,3 \\
\hline
\end{tabular}

Tabla 2. Flujos de financiación entre sectores. Tomado de Vélez: 2006: 115

De este cuadro se destaca que casi la mitad de los recursos de las organizaciones de la sociedad civil provienen de su mismo sector (otras fundaciones empresariales o tal vez cajas de compensación), más que del gubernamental y mucho más que de los mismos organismos internacionales. Esto implica la aparición de proyectos autónomos que sólo a través de la negociación política es posible coordinar a través de adhesiones a tratados internacionales o metas comunes como los objetivos del milenio.

La rendición de cuentas de 34 ONG adscritas a la Federación Antioqueña de ONG, que se presentó en julio de 2006, también planteó que estas 34 organizaciones, gastaron casi el mismo presupuesto que las Secretarías de Desarrollo Social y de Bienestar Social de la Alcaldía de Medellín en el año de 2005 (FAONG:2006). Hay que tener en cuenta que la administración pública representa el cuarto lugar como 
fuente de financiación a través de la contratación pública ${ }^{10}$. La mayor es la proveniente de organizaciones privadas en un $42 \%$. Además tienen altos niveles de cobertura de la población, equiparables a los de las dos secretarías antes mencionadas. Así lo afirman en el informe presentado: "al menos en Antioquia resulta evidente que las ONG no dependen del Estado para subsistir" (FAONG 2006: 3) ${ }^{11}$.

En cuanto a las comunitarias, un poco menos de la mitad no tienen fuentes de financiación, esto implica fragilidad para la ejecución de proyectos, pero también autonomía en cuanto a su recurso humano voluntario. Este carácter autónomo tiene un peso importante, ya que permite cierto ambiente para la deliberación y evitar los conflictos por saber quien queda o no queda contratado sacando algo a su favor. Sin embargo, todavía subsisten algunas prácticas clientelistas que le quitan esa fuerza a la participación informal y voluntaria, además de presentar dificultades para la planeación formal del bienestar en su comunidad.

La tendencia que se puede observar, en que el tercer sector puede ser independiente de la administración pública, pero de todos modos debe sostener relaciones de dependencia con otros sectores que le permiten esta apertura de alternativas para su subsistencia. Esta tendencia convierte a las propuestas en contigentes, en tanto sus resultados no pueden ser coordinados de manera estratégica. Sin embargo también es la oportunidad para la diversificación de ofertas y la generación de coordinaciones territoriales. Si bien existe libertad, esta libertad en el terreno de la competencia por los recursos y la puesta en lo público de visiones y proyectos, genera inevitablemente contigencia, en tanto no existan mecanismos coordinadores de lo global. Existen múltiples centros y esos centros, con múltiples propuestas para el desarrollo (unas de mayor alcance que otras). Ello se puede observar en la desconexión entre las organizaciones con mayor centralidad en ambas redes, por ejemplo en el intercambio de recursos.

\footnotetext{
${ }^{10}$ A pesar de que en las dos redes, las organizaciones estatales sean las que tienen mayor centralidad en las asignaciones de recursos: en la red del sistema de atención a la niñez, de 330 relaciones de intercambio existentes, 107 provienen de organizaciones gubernamentales (Vélez:2006:116); y de 62 relaciones de oferta de recursos (centralidad output), 35 provienen de éste (Brand et al:2007:95). Esto habla de la relevancia del Estado en la planeación y direccionamiento del desarrollo, pero no es suficiente en tanto los recursos restantes (que no son pocos, más de la mitad) están dispersos en múltiples visiones que pueden ser complementarias de manera accidental o yuxtapuestas en el peor de los casos.

${ }^{11}$ Medellín es la capital del departamento de Antioquia.
} 


\begin{tabular}{|c|c|c|l|}
\hline Rank & Punto & No.Org. & Id. \\
\hline 1 & 61 & 6 & Secretaría de Cultura \\
\hline 2 & 47 & 5 & EPM \\
\hline 3 & 46 & 5 & Secretaría de Desarrollo Comunitario \\
\hline 4 & 53 & 3 & Inder \\
\hline 5 & 48 & 3 & Fondo Focus \\
\hline 6 & 56 & 2 & Metrojuventud \\
\hline 7 & 37 & 2 & Compasión Internacional \\
\hline 8 & 35 & 2 & Comfama \\
\hline 9 & 33 & 2 & Secretaría de Bienestar Social \\
\hline 10 & 64 & 2 & Secretaría General \\
\hline
\end{tabular}

Tabla 3. Tabla de organizaciones con mayor centralidad en la financiación Comuna 6 (tomado de Brand et al. 2007:95).

\begin{tabular}{|c|c|c|l|}
\hline Rank & Punto & No.Org. & I d. \\
\hline 1 & 40 & 35 & Icbf \\
\hline 2 & 123 & 32 & Benefactores \\
\hline 3 & 120 & 15 & Alcaldía de Medellín \\
\hline 4 & 113 & 10 & Medellín-secretaría de educación municipal-unidad de calidad \\
\hline 5 & 45 & 9 & Comfama \\
\hline 6 & 48 & 7 & Comfenalco-coordinación de Educación Infantil y Programas Especiales \\
\hline 7 & 144 & 7 & Fundación bancolombia \\
\hline 8 & 183 & 6 & Alcaldía de Medellín-Secretaría de Bienestar Social \\
\hline 9 & 146 & 6 & Fundación Éxito \\
\hline 10 & 174 & 5 & Plataforma Tierra de Hombres \\
\hline
\end{tabular}

Tabla 4. Tabla de organizaciones con mayor centralidad en la financiación Sistema de Ateción a la Niñez Medellín (tomado de Vélez 2006:223)

Sólo coincide Comfama (Caja de Compensación) en los esquemas de los principales financiadores en términos de relaciones de centralidad. Habría que tener en cuenta que Comfama atiende múltiples frentes y no necesariamente hay coincidencia entre la temática de niñez y lo financiado a las organizaciones de la Comuna. No es el único indicador que aparece con estas similares características en ambos estudios (también se pude ver en las centralidades de cooperación e intercambio de información, por ejemplo). Esto denota exclusión mutua y autonomía en la toma de decisiones de dos aspectos del desarrollo (la niñez y el desarrollo comunitario). Estas organizaciones tendrían que tener una estructura de articulación que les permitiera generar acuerdos con respecto a las metas del desarrollo local, sin embargo, al no existir este tipo de espacios formales, se conforman parcelas aisladas de acción, sin una propuesta integral e integrada para lo local. 
No se puede decir que no existen espacios de articulación que congreguen a algunas de estas organizaciones; sin embargo estos espacios de articulación son especializados y no permiten un debate integral sobre las perspectivas. De otro lado, podría denotarse exclusión en la participación de la constitución de las agendas para el desarrollo local, de organizaciones que están por fuera del alcance de estas organizaciones centrales, pero que no detentan la mayoría de recursos del sistema, pues están dispersos en múltiples financiadores.

La independencia sectorial en la toma de decisiones y las centralidades fragmentadas en la financiación por ejemplo, llevan a generar dos tendencias para las organizaciones del Tercer Sector: una que hace que las organizaciones sean competitivas para participar en concursos para la financiación de proyectos; otra que identifica un tipo de organizaciones no competitivas y que trabajan con base en la colaboración y el voluntariado. Esto genera dos formas de autonomías: una que tiene que ver con la abundancia de fuentes o no para el acceso de recursos y de esa forma poder plantearse objetivos propios y no ajenos ( o al revés, en la pobreza de fuentes y la cooptación), la otra si bien escasea de recursos financiaeros para adelantar proyectos, posee un capital social importante que le permite desarrollarlos en el seno de las comunidades. Obviamente la envergadura de los proyectos en estos dos tipos de autonomías es distinta, sin embargo las funcionalidades sociales son distintas: las primeras prestarán servicios sociales, las segundas desarrollarán la capacidad organizativa de las comunidades y ampliarán las posibilidades de resolución de sus problemáticas de manera más o menos autónoma. Sin embargo, la tendencia del mercado a generar recursos para lo social, marca de manera definitiva las dinámicas de interacción, cada vez llevando más a las organizaciones a competir por recursos luego de lograrse cierto avance en la consolidación organizativa comunitaria.

\subsection{Articulación y fragmentación}

La contingencia expuesta anteriormente tiene también consecuencias directas para la búsqueda de espacios de articulación y generación de acciones colectivas. Esto se evidencia en la explosión de una gran cantidad de este tipo de propuestas. Por ejemplo en el Sistema de Atención a la Niñez de Medellín, 102 organizaciones que respondieron a este apartado de la encuesta dijeron pertenecer por lo menos a 162 espacios de articulación reconocidos (Vélez:2006:153). Por lo menos 12 de estos resultan ser los que más convocatoria tienen y los más relevantes para el sistema. De otro lado, existen por lo menos 10 organizaciones que concentran las mayores relaciones de participación en distintos espacios (casi un cuarto de las 
participaciones). Esto habla de una alta concentración en menos de la mitad de las organizaciones de las oportunidades de participación en espacios colectivos o de articulación. Es importante destacar que de los doce espacios de participación más concurridos no todos trabajan directamente el tema de la niñez; los otros son convocados por la administración pública como mecanismos de coordinación de la oferta de programas del gobierno y la definición de metas con respecto a la población; otros son convocados por la sociedad civil y han servido como importantes mecanismos para posicionar la temática en el terreno de lo público.

A pesar de la jerarquía de estos espacios de articulación por su convocatoria e impacto en la problemática, siguen conviviendo con otros 150 espacios en donde se realizan coordinaciones de algún tipo para planear las actividades organizacionales. Esto presenta altos niveles de contingencia en la construcción de perspectivas de desarrollo. La vía para mitigar la contingencia es el acuerdo político, y en este se incluyen las adhesiones a programas de acuerdo global o a través de la legislación vigente especializada en la temática. Se logran acuerdos pero faltan vinculaciones y tal vez ese es el papel de los espacios de articulación más representativos (aunque se ha avanzado, todavía es una tarea pendiente, de largo plazo y con dificultades, las propias de cualquier negociación de carácter político).

Con respecto a la comuna 6 , la tendencia es distinta. Hay unos pocos espacios de mayor relevancia para la comuna como los convocados por el gobierno para realizar planeaciones participativas o generar un buen ambiente para los presupuestos participativos; u otros de carácter civil como las asociaciones de organizaciones (Asocomunal por ejemplo) o la Red de Organizaciones Comunitarias que agrupa a las que tienen un mayor grado de modernización, especialmente. Sin embargo entre las 133 organizaciones sólo se encontraron 110 espacios de articulación (Brand et al:2007: 148), pero sólo 96 están articuladas a estos.

La abundancia de espacios de articulación en el Sistema de Ateción a la Niñez de Medellín, la cual sobre pasa el número de organizaciones encuestadas, en contraste con el menor número de articulaciones, por debajo inclusive del número de organizaciones existentes en la comuna 6 , denota dificultades para que la acción colectiva se presente. Hay dos explicaciones posibles para esto: una, que la tendencia a la articulación se presenta más en organizaciones con un mayor nivel de modernización, o que la articulación entre las comunitarias al tener un carácter más localizado, no logren congregarse alrededor de espacios un poco más abstractos como los comunales y lo hagan en el seno de sus localidades o barrios, mas no con pretensiones comunales. Este último argumento puede tener más peso 
que el primero, dado que las relaciones entre organizaciones tienden a sectorizarse en punto focales, en los cuales existen dos o tres organizaciones fortalecidas que congregan las organizaciones comunitarias de los barrios más próximos, más que de toda la comuna. Los únicos espacios que logran la convocatoria comunal, son los citados por el gobierno prácticamente. Se puede presentar pues, una tendencia a la localización de lo comunitario, más acá de las fronteras establecidas por la división político administrativa del municipio.

De estos datos se pueden destacar las tendencias hacia una articulación que es efecto de la contingencia, pero que a su vez genera más contingencia para la planeación de un horizonte común en lo local. Esas formas de articulación de pueden observar como tendencias a la búsqueda de formas de coodinación adecuadas que permitan en varios niveles la generación de acuerdos políticos para generar resultados más importantes en la implementación de proyectos. La articulación se presenta pues fragmentada en estos términos, pero con tendencia a la ubicación de varios centros desde los cuales se toman decisiones importantes.

La otra tendencia es a la articulación local desde las organizaciones comunitarias, lo cual no coincide por lo regular con las planeaciones participativas propuestas por el gobierno municipal a partir de la división político-administrativa. Mientras que las comunidades planean entre sus barrios vecinos, el gobierno pide planear integralmente con todos los miembros de la comuna: aquí, al escasear los recursos, entran en competencia para la priorización de proyectos que entren dentro de los presupuestos de carácter participativo.

La tendencia a la planeación y articulación local y los múltiples centros de decisiones articuladas que se conforman en la gestión temática (o poblacional: la niñez), apenas comienzan a crusarse a través de reuniones y propuestas lanzadas como por ejemplo la del Observatorio de Niñez de la ciudad o algunas experiencias de la Red Antioqueña de Niñez, trabajadas en conjunto con algunas organizaciones comunitarias, pero en general siguen siendo dos tendencias a la atención al desarrollo distintas y no articuladas. Sin embargo hay una tendencia a la búsqueda de unos y otros para la negociación.

\section{Algunas conclusiones}

Sobre tendencias analíticas que pueden ser útiles para caracterizar el Tercer Sector de manera que permita observar otras problemáticas presentes en el mismo:

Se evidencian algunas tendencias que permiten una descripción del estado actual de las organizaciones civiles y sus múltiples formas de configuración: 
Hay una tendencia a la separación de tipos de organizaciones especializadas en la intervención territorial y otras a la especialización temática. Estas propuestas tienden a la divergencia. Esto implica una separación de dos subsectores visibles, dentro del Tercer Sector, sin contemplar otro tipo de posibilidades como las organizaciones cooperativas o mutualistas por ejemplo que podrían tener rasgos de ambos tipos de relación y nuevas características.

Hay otra tendencia también a la construcción de nuevos subsectores a partir de la separación relacional de organizaciones con altos índices de modernización, otras de carácter informal, y aquellas que se presentan como híbrido de de ambas características. Debido a la contingencia generada por la acción de estas organizaciones relativamente autónomas, aparece la gestión como forma de reducir contingencia, pero esas estrategias se presentan de dos formas: unas se especializan en la gestión de recursos económicos a través de la competencia en la presentación de proyectos a organismos financiadores; otras lo hacen a través de prácticas como el empoderamiento para la participación, lo cual genera sinergias y capital humano útil a la organización. Esto lleva inclusive a la diferenciación en los ámbitos de acción: mientras las gestoras de recursos económicos y con altos índices de modernización buscan la atención de temáticas especializadas como la niñez, buscando amplias coberturas o la implementación de programas piloto posibles de replicar; las organizaciones informales se interesan más en la atracción de voluntades a través de sus propuestas políticas, ideológicas o prácticas, de personas que tengan cierta afinidad identitaria a partir de compartir el mismo barrio o sector, la existencia de problemáticas cotidianas comunes o la afinidad en intereses políticos e ideológicos.

La contigencia genera una tendencia más y es hacia la aparición de múltiples centros para la toma de decisiones sobre el desarrollo: los centros propuestos por múltiples redes de coordinación y deliberación, además de las decisiones gubernamentales que también se yuxtaponen en algunos casos entre ellas mismas y con programas de otros sectores y finalmente los centros de las mismas fundaciones patrocinadas por organizaciones productivas. Estos múltiples centros tienden a la fragmentación de un horizonte común, el cual sólo es posible intervenir, en esta lógica, desde instituciones acordadas o preexistentes, expresas en leyes o acuerdos internacionales.

Esta tendencia a la fragmentación y a la aparición de múltiples centros de toma de decisiones, también se expresa en la gran cantidad de espacios de articulación existentes, pero que al generar esta multiplicidad de centros de toma de decisiones, permite apreciar la paradoja de la articulación fragmentada. Nuevamente la libertad 
tiene su precio, que es pagado con complejos mecanismos y niveles de articulación y coordinación social que tienen que establecerse en debates y deliberaciones que son de largo plazo.

Sobre tendencias de la investigación en el Tercer Sector:

El análisis de redes sociales, además de los parámetros de medición de la sociometría, en combinación con teorías intermedias genera nuevas categorías analíticas propias del análisis relacional y ausente de otros tipos de investigación y análisis. El Tercer Sector en Latinoamérica es un sector vasto, que presenta la oportunidad de generar lecturas distintas acerca de la construcción del desarrollo por una vía alterna al de las políticas públicas o las posibilidades del mercado libre. Estas alternativas pueden ser motores de innovación que permitan refrescar, renovar o reformar las formas de construcción de las políticas públicas para lograr una consonancia con las propuestas del Tercer Sector o inclusive el sector productivo que también tiene sus propias lógicas relacionales.

Agradecimientos:

Al grupo de Análisis de Redes Sociales con el que he trabajado desde la Universidad de Antioquia (Edinson Brand, Diana Castaño, Patricia Trujillo, Henry Gómez y Juan Esteban Rodríguez), pues sin ellos hubiese sido imposible construir este artículo, como tampoco sin el apoyo y las discusiones generadas dentro del Observatorio de Niñez de Medellín y sus organizaciones patrocinadoras y ejecutoras: ICBF, Alcaldía de Medellín, CINDE, Universidad de Antioquia, Fondo para la Acción Ambiental y UNICEF. Especiales agradecimientos a Diego Flórez, Helena Torres y Adriana Calle, quienes también participaron de las investigaciones realizadas.

\section{Bibliografía}

Brand, Edinson et al. (2007). Estructura de Redes Interorganizacionales: Análisis de Redes Sociales Comuna 6 Medellín. Medellín: Corporación Picacho con Futuro, CEO Universidad de Antioquia. Tomado de: http://revistaredes.rediris.es/webredes/novedades/FINAL.pdf el 13 de marzo de 2007.

Cravacuore, Daniel, Sergio Ilari y Alejandro Villar (2004). La articulación en la gestión municipal. Bernal (Argentina): Universidad Nacional del Quilmas.

Elster, Jon (1991). El Cemento de la Sociedad. Barcelona: Gedisa

Federación Antioqueña de ONG (2006). Rendición Social de Cuentas de 34 Organizaciones Antioqueñas. Sin editar, disponible en la Federación.

Forni, P. y E.M. Longo (2004). "Las respuestas de los pobres a la crisis: Las redes de organizaciones comunitarias y la búsqueda de soluciones a los problemas de las áreas periféricas de Buenos Aires", Redes. Revista hispana para el análisis de redes sociales, 6 (6), <http://revista-redes.rediris.es >. 
González Bombal, Inés y Rodrigo Villar (2003). Organizaciones de la sociedad civil e incidencia en políticas públicas. Buenos Aires: Libros del Zorzal.

Knoke, David (1986). "Association and Interest Groups", Annual Review of Sociology, Vol. 12, 1-21

Lazega, Emmanuel (2004). "Racionalidad, disciplina social y estructura", Redes. Revista hispana para el análisis de redes sociales, 5 (6), <http://revistaredes.rediris.es>.

Le Galès, Patrick, Mark Tatcher (1995). Les Réseaux de politique Publique. Paris: L'Harmattan.

Natal Martínez, Alejandro (1998). How state founds impact NGO's capacity to foster people's participation. Paper prepared for the McNamara Fellowship. The Wolrd Bank. Sin editar.

Olson, Mancur (1965). The logic of collective action : public goods and the theory of groups. Cambridge, Mass: Harvard University.

Pfeffer, Jeffrey y Salancik, Gerald (1978). The external control of organizations: A resource dependence perspective. New York: Harper \& Row, publishers.

Palacio, Dolly, Rafael Hurtado y Leonardo Garavito (2003). "Redes Socioambientales en tensión: El caso de la gestión ambiental de los humedales de Bogotá", Redes. Revista hispana para el análisis de redes sociales, 4 (6), <http://revista-redes. rediris.es $>$.

Porras Martínez, José Ignacio (2000). "Empresarios, Acción Colectiva y Política Agrícola en Bolivia y Perú. Una aproximación desde el análisis de los policy networks". Tomado de: http://revistaredes.rediris.es/webredes/textos/Empregr.pdf el 6 de marzo de 2006.

Rofman, Adriana y Alejandro Villar (comp.) (2006). Desarrollo Local: Una Revisión Crítica al Debate. Buenos Aires: Espacio Editorial.

Salamon, Lester M., Helmut K. Anheier, Regina List, Stefan Toepler, S. Wojciech Sokolowski y Colaboradores (1999). La Sociedad Civil Global: Las Dimensiones del Sector no lucrativo. (Proyecto de Estudio comparativo del sector no lucrativo de la Universidad J onhs Hopkins). Madrid: Fundación BBVA. Traducción de la versión en Inglés: Global Civil Society: Dimensions of the non-profit sector. Baltimore: Johns Hopkins Center for the civil society studies. Un resumen de este estudio puede encontrarse en:

www.plataforma.uchile.cl/fg/semestre1/2004/asocia/modulo1/clase3/doc/estudio. $\underline{\mathrm{pdf}}$

Sulbrandt, J osé; Lira Ricardo y Américo Ibarra (2001). “Redes interorganizacionales en la administración pública". En: Reforma y Democracia. Revista del Clad (Centro latinoamericano de administración para el desarrollo), No. 21. Octubre. Caracas.

Turk, Herman (1970). "Interorganizational Networks in Urban Society: Initial Perspectives and Comparative Research", American Sociological Review, Vol. 35, No. 1, Febrero, pp. 1-19. 
Vélez Cuartas, Gabriel. (2006). Sistema Organizacional de Atención a la Niñez de Medellín: Estructura relacional de 128 organizaciones gubernamentales y de la sociedad civil. Medellín: ICBF, Alcaldía de Medellín, Cinde, Universidad de Antioquia; Observatorio de Niñez del Municipio de Medellín. Tomado de: http://revista-redes.rediris.es/webredes/textos/sistema.pdf, marzo de 2006. 